\title{
El tratamiento con pregabalina no redujo la intensidad de la lumbociatalgia aguda 0 crónica
}

Treatment with pregabaline did not reduce intensity of acute or chronic sciatica pain

Mathieson S. y col. N. Engl J Med 2017;376:1111-20.

\section{Objetivos}

Determinar si la pregabalina es efectiva para aliviar el dolor del miembro inferior afectado en la lumbociatalgia aguda y crónica (resultado primario); y determinar el efecto de su uso sobre el dolor lumbar, el grado de discapacidad, la calidad de vida, el ausentismo laboral y la utilización de servicios de salud (resultados secundarios).

\section{Diseño, lugar y pacientes}

Ensayo clínico aleatorizado, doble ciego, controlado con placebo, realizado en 47 centros médicos ubicados en Nueva Gales del Sur, Australia. Se incluyeron 209 pacientes mayores de 18 años, entre 2013 y 2015, con lumbociatalgia de más de una semana y menos de un año de evolución. Los pacientes fueron asignados al grupo pregabalina $(n=108)$, partiendo de $150 \mathrm{mg} /$ día y ajustando la dosis hasta un máximo de $600 \mathrm{mg} / \mathrm{día}$, o al grupo placebo $(\mathrm{n}=101)$, por ocho semanas. Durante el ensayo, los pacientes podían recibir tratamientos adicionales como kinesioterapia y otros analgésicos, excluyendo aquellos que pudieran actuar como coadyuvantes de la pregabalina.

\section{Medición de resultados principales}

La efectividad de la pregabalina fue evaluada en términos del promedio de reducción de la intensidad del dolor en el miembro inferior afectado, utilizando una escala de dolor de 0 a 10 (0 indica que no hay dolor y 10 el peor dolor posible). La diferencia clínicamente importante se observa a partir de 1,5 puntos en la escala, medida a las 8 y 52 semanas de comienzo del estudio. Se realizó un análisis de subgrupo para evaluar si los pacientes con dolor de características neuropáticas, (identificadas por el cuestionario "PainDETECT"), presentaban mejor respuesta al tratamiento.

El análisis se realizó por intención de tratar.

\section{Resultados principales}

Los resultados principales se muestran en la tabla 1. No se encontraron diferencias estadísticamente significativas en los resultados secundarios.

En el análisis de subgrupo, la presencia de dolor de características neuropáticas no modificó el efecto del tratamiento a las ocho semanas $(p=0,13)$.

No se registraron diferencias significativas en efectos adversos graves entre los grupos. Los efectos adversos leves a moderados, principalmente mareos, fueron más frecuentes en el grupo pregabalina.

Tabla 1. Efectividad de la pregabalina (promedio de reducción en la intensidad de dolor escala de 0 a 10).

\begin{tabular}{|c|c|c|c|c|}
\hline Resultados y tiempo de la evaluación & $\begin{array}{c}\text { Grupo pregabalina } \\
(n=108)\end{array}$ & $\begin{array}{c}\text { Grupo placebo } \\
(n=101)\end{array}$ & Diferencia de medias ajustada (IC 95\%) & Valor $\mathbf{p}$ \\
\hline Semana 0 & $6,3 \pm 1,8$ & $6,1 \pm 1,9$ & & \\
\hline Semana 8 & 3,7 & 3,1 & $0,5(0,2$ a 1,2$)$ & 0,19 \\
\hline Semana 52 & 3,4 & 3,0 & $0,3(-0,5$ a 1,0$)$ & 0,46 \\
\hline
\end{tabular}

IC95\%: intervalo de confianza del 95\%.

\section{Conclusiones}

El tratamiento con pregabalina, administrada durante ocho semanas, no redujo significativamente la intensidad de la lumbociatalgia ni otros resultados de interés, al comparla contra pla- cebo; y se asoció a un incremento de efectos adversos.

Fuentes de financiamiento: National Health and Medical Research Council of Australia. Las capsulas fueron provistas por Pfizer Australia, sin costo.

\section{Comentario}

Distintas guías consideran que el rol de los anticonvulsivantes (ej. gabapentina, topiramato, pregabalina) es a lo sumo modesto o nulo en el manejo del dolor lumbar asociado a radiculopatía ${ }^{1,2}$. Sin embargo algunas ofrecen un ciclo de estas drogas como opción de manejo cuando el dolor se considera neuropático y no responde a otras estrategias (ej. AINE) ${ }^{3,4}$. Al margen de esto la evidencia que respalda su efectividad, y en particular para pregabalina, resulta inconsistente y escasa ${ }^{5}$. En el estudio que comentamos el criterio de selección de los pacientes fue basado en las características clínicas de la lumbociatalgia, y se realizó un ajuste de dosis individual, similar a lo que ocurre en la práctica médica.

\section{Conclusiones del comentador}

El tratamiento con pregabalina no redujo significativamente la intensidad del dolor en la pierna con ciatalgia, no mejoró significativamente otros resultados, y aumento la incidencia de efectos adversos, lo que sugiere que no debe generalizarse su uso a todos los tipos de dolor que cumplan características neuropáticas. Al menos por ahora, la pregabalina parece no constituir una opción válida para el tratamiento de la lumbociatalgia.

Sofía Victoria Gürtler y Gabriel Villalón [ Servicio de Medicina Familiar y Comunitaria del Hospital Italiano de Buenos Aires. sofia.gurtler@hospitalitaliano.org.ar ]

Gürtler S, y Villalón G. El tratamiento con pregabalina no redujo la intensidad de la lumbociatalgia aguda o crónica. Evid Act Pract Ambul. 2017;20(2):39. Comentado de: Mathieson S. y col. Trial of Pregabaline for Acute and Chronic Sciatica. N. Engl J Med 2017;376:1111-20. PMID: 28328324.

Referencias

1. Chou R, y col. Diagnosis and treatment of low back pain: a joint clinical practice guideline from the American College of Physicians and the American Pain Society. Ann Intern Med. 2007:147(7):478-491.

2. Goertz M, y col. Adult Acute and Subacute Low Back Pain. Institute for Clinical Systems Improvement [Internet]. Updated November 2012. Disponible en: https://www.icsi.org/ guidelines_more/catalog_guidelines_and_more/catalog_guidelines/catalog_musculoskeletal_guidelines/low_back_pain/

3. National Institute for Health and Care Excellence. Neuropatic pain in adults: pharmacological management in non-speciallist settings (en línea). 2013. NICE guidelines CG173. Disponible en: https://www.nice.org.uk/guidance/cg173/resources/neuropathic-pain-in-adults-pharmacological-management-in-nonspecialist-settings-pdf-35109750554053 4. National Institute for Health and Care Excellence. Sciatica (lumbar radiculopathy). Clinical knowledge summaries (en línea). 2015. Disponible en: https://cks.nice.org.uk/ sciatica-lumbar-radiculopathy\#!scenario (último acceso agosto 2017).

5 . Shanthanna $\mathrm{H}$, y col. Benefits and safety of gabapentinoids in chronic low back pain: A systematic review and metaanalysis of randomized controlled trials. PLoS Med 2017;14(8): e1002369. https://doi.org/10.1371/journal. pmed.1002369 